\section{Análise dos dados dos Centros de Controle de Intoxicação do Rio de Janeiro, Brasil, como subsídio às ações de saúde pública}

\author{
Analysis of data from Poison Control Centers in \\ Rio de Janeiro, Brazil, for use in public health \\ activities
}

\footnotetext{
${ }^{1}$ Instituto Nacional de Controle de Qualidade em Saúde, Fundação Oswaldo Cruz, Rio de Janeiro, Brasil. 2 Escola Nacional de Saúde Pública Sergio Arouca, Fundação Oswaldo Cruz, Rio de Janeiro, Brasil.

Correspondência R. F. Presgrave Departamento de Farmacologia e Toxicologia Instituto Nacional de Controle de Qualidade em Saúde, Fundação Oswaldo Cruz.

Av. Brasil 4365, Rio de Janeiro, $R J$ 21045-900, Brasil. rosaura.presgrave@incqs. fiocruz.br
}

\begin{abstract}
Health information systems should be sources of reliable and valid data that can be used for surveillance to reduce morbidity and mortality. Unintentional poisoning is highly relevant to public health, since it is the most frequent cause of emergency pediatric care. Information on such events is recorded in Poison Control Centers (PCCs). The current study analyzed the records on unintentional poisoning with household cleaning products in two PCCs in the State of Rio de Janeiro, Brazil, in 2000-2002. The variables analyzed were gender, age, cause of the accident, exposure route, toxic agent, and outcome. In the 2,810 records studied, boys four years and younger were the most vulnerable group and oral ingestion was the most common exposure route. However, analysis of the data on the toxic agent, cause, and outcome showed that the data currently recorded in the PCCs are inconsistent. It is necessary to review the definitions used by the PCCs in order for the resulting data to effectively support public health measures and health surveillance policies.
\end{abstract}

Poisoning; Poison Control Centers; Information Systems; Health Surveillance
Rosaura de Farias Presgrave 1

Luiz Antônio Bastos Camacho 2

Maria Helena Simões Villas Boas 1

\section{Introdução}

Conforme está descrito na Lei $n^{o}$. 8.080, de 19 de setembro de 1990, a Vigilância Sanitária é um dos campos de atuação do Sistema Único de Saúde (SUS) e está definida como "um conjunto de ações capaz de eliminar, diminuir ou prevenir riscos à saúde". Deste modo, a Vigilância Sanitária é importante pelo caráter preventivo das suas ações, dentre as quais está a identificação de fatores de risco à saúde humana associados aos produtos consumidos pela população 1 .

A determinação do risco de um produto é dada pela avaliação das propriedades tóxicas das substâncias químicas (seja um medicamento, um poluente ambiental ou um produto industrial) e pelas condições da exposição humana. As informações sobre população de risco, vias e magnitude da exposição sob várias condições são usadas na avaliação do risco de efeitos adversos em condições plausíveis de exposição 2,3. Fatores inerentes ao produto como cor, odor e aspecto podem afetar o risco de exposição.

Para que medidas preventivas possam ser implementadas, é necessário que sistemas de informação em saúde identifiquem os efeitos adversos determinados por um agente tóxico (toxicovigilância) que, muitas vezes, são observados somente após a comercialização do produto, seja um produto novo, modificação de uma formulação ou da finalidade de uso. O conceito de toxi- 
covigilância abrange não somente a detecção do efeito adverso, mas a validação e o acompanhamento dos casos clínicos relacionados à exposição humana aos agentes tóxicos 4 .

A Organização Mundial da Saúde (OMS) define Sistema de Informações em Saúde (SIS) como "um conjunto de componentes que atuam de forma integrada, por meio de mecanismos de coleta, processamento, análise e transmissão da informação necessária e oportuna para implementar processos de decisões no Sistema de Saúde" 5 (p. 4). Os sistemas de informação em saúde são essenciais nas ações de saúde pública para reduzir morbidade e mortalidade e para melhorar a saúde da população. Por isso, as informações geradas devem ser confiáveis e válidas, sendo a qualidade dos registros um elemento essencial para aqueles atributos 6 .

As intoxicações, principalmente as não intencionais, constituem a principal causa de atendimento de emergência pediátrica. No Brasil, os dados sobre intoxicações são disponibilizados nas publicações anuais do Sistema Nacional de Informações Tóxico-Farmacológica (SINITOX; http://www.fiocruz.br/sinitox), que compila as informações dos 36 Centros de Controle de Intoxicações (CCIs) localizados em 19 estados e no Distrito Federal. No período de 1994 até 2003, foram notificados 694.538 casos de intoxicação humana no Brasil, sendo 291.783 casos (42\%) com produtos sujeitos à Vigilância Sanitária (medicamentos, saneantes, cosméticos, alimentos). Os medicamentos e os produtos saneantes (pesticidas domésticos, raticidas e domissanitários) são as principais causas de intoxicação, sendo que os casos não intencionais ocorrem em maior número com produtos saneantes.

As informações sobre intoxicações são detalhadas em fichas de notificação existentes nos CCIs, preenchidas durante o atendimento das ligações telefônicas, provenientes dos estabelecimentos de saúde ou da população, solicitando orientação para o tratamento de intoxicados. A primeira versão da Ficha de Notificação e de Atendimento e seu respectivo Manual de Preenchimento foram divulgados pela Fundação Oswaldo Cruz (FIOCRUZ), em 1997, tendo sido a última versão atualizada em 2001 por representantes do SINITOX e da Rede Nacional dos Centros de Controle de Intoxicações 7.

A avaliação dos dados diários obtidos em tempo real e contínuo permite aos CCIs identificar problemas antes de estes terem sido reconhecidos por autoridades de saúde, enquanto que a avaliação dos dados agregados existentes nos CCIs contribui para a avaliação do risco, demonstrando os dados de exposição humana. Pa- ra atender a essas necessidades, os CCIs precisam verificar continuamente a qualidade do processo de geração e análise dos dados 8,9,10,11.

Para subsidiar a implementação de medidas preventivas, seja junto à população exposta ou aos fabricantes, bem como as intervenções nas normas de registro/notificação de produtos no Ministério da Saúde, há limitações nos dados, inclusive em variáveis críticas como a identificação do produto tóxico e a causa do evento.

Considerando que somente a utilização e avaliação dos dados existentes em qualquer sistema de informação permitem detectar erros ou inconsistências, contribuindo assim para a melhoria da qualidade das informações, temos como objetivo deste trabalho analisar a qualidade do preenchimento das fichas de notificação de intoxicação não intencional com produtos saneantes domissanitários registradas nos dois CCIs do Estado do Rio de Janeiro, no período de 2000 a 2002, a fim de caracterizar a população vulnerável e o curso clínico destes eventos.

\section{Metodologia}

Foram analisados os dados das fichas de notificação 7 referentes às intoxicações não intencionais com produtos saneantes domissanitários, ocorridas em residências na área urbana, no período de 2000 a 2002 existentes no Centro de Informação e Assistência Toxicológica do Rio de Janeiro (CIAT-RJ), localizado no Hospital Universitário Clementino Fraga Filho da Universidade Federal do Rio de Janeiro e no CCI de Niterói (CCI-n), localizado no Hospital Universitário Antônio Pedro da Universidade Federal Fluminense.

Foram consultadas 13.429 fichas tendo sido selecionadas 2.200 referentes à intoxicação não intencional com produtos saneantes domissanitários e 610 fichas de intoxicação com domissanitário, mas que estavam classificadas em outras categorias. Foram excluídas deste estudo 34 fichas, pois, apesar de estarem classificadas como intoxicação por domissanitário ou pesticida doméstico, o produto envolvido era cosmético (26) ou produto de uso veterinário (8).

As variáveis analisadas foram: idade e sexo do acidentado, causa do acidente (foram selecionadas apenas as fichas em que estavam assinaladas as circunstâncias de acidente individual ou acidente coletivo), via de exposição, agente tóxico (classificação e identificação do produto) e evolução do caso. As observações descritas na ficha, também foram analisadas. A validade dos dados não foi verificada devido à impossibilidade de comparar os dados dos registros com referências externas 6 . 
Os dados foram codificados, digitados em planilha do Excel (Microsoft Corp., Estados Unidos) e analisados pelo Epi Info 6.04 (Centers for Disease Control and Prevention, Atlanta, Estados Unidos).

Este estudo foi aprovado pelo Comitê de Ética em Pesquisa Humana da FIOCRUZ (protocolo 227/03).

\section{Resultados}

\section{Idade e sexo}

A variação da faixa etária dos indivíduos era de 18 dias a 91 anos, sendo a idade média de 8,9 e o desvio padrão 15,93. O grupo mais afetado era caracterizado por meninos na faixa etária de $0 \mathrm{a}$ 4 anos. A idade era ignorada em 48 casos $(1,7 \%)$ e o sexo em 25 casos $(0,9 \%)$.

\section{Produto}

Do total de fichas, somente em 487 (17,3\%) o produto foi identificado corretamente, com o nome comercial. Em 2.323 fichas $(82,7 \%)$, a identificação do produto não era adequada: 175 fichas $(6,2 \%)$ estavam sem preenchimento do dado, sendo que em apenas 7 fichas, o notificante declarou que o produto era ignorado, e 2.148 fichas $(76,4 \%)$ estavam com a informação incompleta, conforme as situações descritas a seguir. Em
1.783 fichas foi identificada apenas a categoria do produto (sem marca e/ou complemento do nome), por exemplo inseticida, detergente, água sanitária. Em 275 fichas o produto foi identificado apenas pela marca e em 90 fichas $(3,2 \%)$, o campo foi preenchido com a descrição do princípio ativo.

\section{Classificação do produto}

A classificação do produto não estava preenchida em 60 fichas $(2,1 \%)$ e a classificação era ignorada em 5 fichas $(0,2 \%)$.

Foram constatadas 610 fichas com outra classificação, sendo 81,3\% classificadas como "produto químico industrial”, e destes, mais da metade eram detergentes (Tabela 1). Quase 10\% não tinham informação sobre a classificação.

\section{Via de exposição}

A informação sobre a via de exposição foi obtida em quase todas as fichas, sendo a via oral a mais freqüente $(90,4 \%)$, seguida da via inalatória $(4,3 \%)$, cutânea $(2,4 \%)$, ocular $(2 \%)$ e outras $(0,4 \%)$. A informação estava ausente em 16 fichas $(0,6 \%)$.

\section{Causa do acidente}

Em 1.241 fichas (44,2\%) não havia nenhum tipo de informação que possibilitasse a identificação

Diferentes denominações atribuídas a produtos saneantes pelos Centros de Controle de Intoxicações (CCls) do Rio de Janeiro, Brasil, 2000 a 2002.

\begin{tabular}{|c|c|c|c|c|c|}
\hline \multirow[t]{2}{*}{ Classificação pelos $\mathrm{CCls}$} & \multicolumn{5}{|c|}{ Categoria de produto saneante domissanitário } \\
\hline & Detergentes & Antimicrobianos & Desinfestantes & Corrosivos & Total \\
\hline Produto químico industrial & 256 & 136 & 55 & 49 & 496 \\
\hline Sem classificação & 18 & 21 & 17 & 4 & 60 \\
\hline Agrotóxico agrícola & - & - & 14 & - & 14 \\
\hline Outros & 2 & 1 & 9 & - & 12 \\
\hline Medicamentos & - & 2 & 7 & - & 9 \\
\hline Ignorado & - & 1 & 4 & - & 5 \\
\hline Droga de abuso & 3 & - & - & - & 3 \\
\hline Limpadores & - & 3 & - & - & 3 \\
\hline Derivados de petróleo & 1 & 1 & - & - & 2 \\
\hline Domissanitário/Produto químico industrial & 2 & - & - & - & 2 \\
\hline Cosmético & - & - & - & 1 & 1 \\
\hline Fumigante & - & - & 1 & - & 1 \\
\hline Medicamento/Pesticida doméstico & - & - & 1 & - & 1 \\
\hline Produto veterinário & - & - & 1 & - & 1 \\
\hline Total & 282 & 165 & 109 & 54 & 610 \\
\hline
\end{tabular}


da causa do acidente. Entretanto, em 1.569 fichas $(55,8 \%)$ havia a citação dos prováveis fatores determinantes do acidente na anotação feita pelo atendente do CCI: produto ao alcance da criança; armazenamento em frasco inadequado causando confusão com refrigerantes, água, alimentos, medicamentos e cosméticos; ocorrência do acidente durante a utilização do produto; uso indevido do produto; uso de utensílio de cozinha para medir produtos de limpeza; mistura de produtos; confusão do aspecto do produto com medicamento ou alimento.

\section{Evolução}

Em 1.018 fichas (36,2\%) não havia informação do desfecho do caso (825 estavam em branco e 193 era ignorado).

Em $62,9 \%$ das fichas em que o desfecho estava em branco, o atendente havia descrito que o paciente havia recebido alta hospitalar.

Uma das opções existentes na ficha de notificação para a evolução dos casos é a ocorrência de seqüelas, mas esta não foi assinalada em nenhum dos casos estudados, nem mesmo quando o produto envolvido era corrosivo.
Na Tabela 2 estão demonstradas outras distorções constatadas ao se comparar a observação descrita na ficha e a evolução assinalada.

\section{Discussão}

Como nas ações de vigilância em saúde em geral, a informação também é um elemento crucial para o desempenho da Vigilância Sanitária. Precisar as limitações do sistema de informação e seus determinantes permite dimensionar as implicações nas decisões em saúde tanto no atendimento individual da intoxicação como em nível coletivo, pela análise da freqüência global e por subgrupos de variáveis de interesse 6 .

A análise dos registros existentes nos CCIs demonstrou baixo percentual de respostas "ignorados/brancos” para idade, sexo e via de exposição, permitindo caracterizar adequadamente a população mais vulnerável aos eventos de intoxicação não intencional com produtos de limpeza domésticos como sendo crianças menores de cinco anos de idade, situação semelhante à de outros países 12,13,14,15. Entretanto, a descrição do curso da intoxicação não está sendo realizada de maneira satisfatória uma vez que o agente tóxico e

\section{Tabela 2}

Freqüência das situações ocorridas em cada um dos desfechos assinalados nas fichas de notificação dos Centros de Controle de Intoxicações (CCls) do Rio de Janeiro, Brasil, 2000 a 2002.

\begin{tabular}{|c|c|c|c|c|c|c|c|c|}
\hline \multirow[t]{2}{*}{ Observação descrita na ficha } & \multicolumn{8}{|c|}{ Evolução preenchida } \\
\hline & Cura & $\begin{array}{l}\text { Cura não } \\
\text { confirmada }\end{array}$ & Seqüela & Óbito & $\begin{array}{l}\text { Óbito por } \\
\text { outra causa }\end{array}$ & Outro & Ignorado & $\begin{array}{c}\text { Em } \\
\text { branco }\end{array}$ \\
\hline Alta & 1.145 & 54 & - & - & - & 24 & 6 & 519 \\
\hline Alta provável & 35 & 41 & - & - & - & 1 & 3 & 24 \\
\hline Assintomático & 185 & 8 & - & - & - & 9 & 1 & 77 \\
\hline Com sintomas & 5 & 1 & - & - & - & - & - & 2 \\
\hline Em observação & 1 & - & - & - & - & - & - & 2 \\
\hline Em tratamento & 3 & - & - & - & - & - & - & 1 \\
\hline Evoluiu bem & 3 & 1 & - & - & - & - & - & - \\
\hline Internado & 3 & 1 & - & - & - & - & - & 2 \\
\hline Em branco & 14 & 3 & - & - & - & - & 9 & 29 \\
\hline Não intoxicação & 1 & 1 & - & - & - & 1 & - & 2 \\
\hline Notificação & - & - & - & - & - & - & - & 1 \\
\hline Outro estado & 1 & 2 & - & - & - & 8 & 6 & - \\
\hline Paciente não localizado & 55 & 129 & - & - & - & 6 & 161 & 150 \\
\hline Sem dados & - & - & - & - & - & - & 2 & 3 \\
\hline Sem relação & - & - & - & - & - & - & - & 1 \\
\hline Saiu à revelia & 7 & 9 & - & - & - & 19 & 2 & 6 \\
\hline Solicitação de informação & 2 & - & - & - & - & 1 & - & 2 \\
\hline Transferido & 1 & 1 & - & - & - & 2 & 3 & 4 \\
\hline Óbito & - & - & - & 9 & - & - & - & - \\
\hline Total & 1.461 & 251 & 0 & 9 & 0 & 71 & 193 & 825 \\
\hline
\end{tabular}


a causa não foram identificados em grande parte dos casos analisados.

Além da determinação da população de risco, é de grande importância para a Vigilância Sanitária a identificação do produto pelo nome e marca. O Decreto $n^{\circ}$. 79.094/77 16 define como nome, "a designação do produto, para distinguílo de outros, ainda que do mesmo fabricante ou da mesma espécie, qualidade ou natureza" e marca, o "elemento que identifica uma série de produtos de um mesmo fabricante ou que os diferencie dos produtos de outros fabricantes". De um modo geral, as empresas produtoras identificam os produtos pela categoria (finalidade de uso) [ex.: detergente lava-louças], marca [ex.: SOL] e às vezes, complemento de marca [ex.: limão]. Deste modo, existem no mercado duas situações: a existência de várias marcas para uma mesma categoria de produto e, a mesma marca sendo usada em diferentes categorias de produtos, implicando composições e princípios ativos distintos.

Foi demonstrado que em cerca de $83 \%$ dos casos, o produto não foi corretamente identificado, impedindo que ações de vigilância sanitária relacionadas ao produto - como, por exemplo, inspeção à indústria, coleta do produto para verificar se satisfaz às normas de segurança preconizadas para aquela categoria, ou até mesmo revisão das normas específicas que regulamentam aquele produto - pudessem ter sido realizadas. $\mathrm{O}$ fato de identificar apenas a marca também é crítico para os CCIs, uma vez que, para a orientação dos primeiros-socorros, principalmente em casos de ingestão, é imprescindível a identificação da substância ingerida 12 .

A legislação sanitária brasileira obriga que conste nos rótulos dos produtos saneantes domissanitários a orientação para que a população leve o rótulo ou embalagem do produto quando necessitar de socorro médico 17,18,19,20,21,22,23. Deste modo, os responsáveis pelo atendimento nos CCIs devem obter esta informação dos notificantes, garantindo a identificação correta do agente tóxico 14 .

Foram constatados também registros em que os produtos foram denominados pelo princípio ativo e outros, onde apesar de não constar o nome do produto, havia a descrição do princípio ativo. Isto sugere que o notificante provavelmente informou qual era o produto envolvido naquele caso, mas o responsável pelo atendimento registrou apenas o dado considerado relevante para a orientação dos primeiros-socorros. Isto pode ser mais um exemplo de que apesar de a ficha de notificação ter sido padronizada, esta é preenchida apenas com as informações de interesse imediato para o atendente, comprometendo a qualidade dos dados para fins de vigilância 24 .
A classificação dos produtos foi outro ponto crítico desta análise, que talvez possa ser justificado pela falta de consenso nas definições adotadas pelos CCIs, como por exemplo, o caso dos produtos químicos. Estes podem ser fabricados para uso doméstico (saneantes, medicamentos, cosméticos) ou uso profissional (industrial, hospitalar ou outras entidades especializadas). Portanto, preconizar a classificação do agente tóxico pela substância envolvida, independente do seu uso, pode levar a distorções sobre a realidade do panorama das intoxicações e gerar equívocos na interpretação dos dados, principalmente pelos gerenciadores do risco. A descrição das categorias existentes no Manual de Preenchimento das fichas de notificação permite equívocos na classificação, uma vez que ora foi utilizado como critério a finalidade de uso e ora, a substância química. O álcool é um bom exemplo disto, uma vez que segundo o manual, pode ser classificado como produto químico industrial ou droga de abuso. Nos casos de ingestão de álcool, esta informação pode estar relacionada a um produto químico industrial, um produto de limpeza (saneante) ou a uma bebida, dando uma conotação diferente ao caso. Para a orientação dos primeiros-socorros isso também é crucial uma vez que dependendo da finalidade de uso, a concentração do princípio ativo também difere, influenciando na severidade do caso.

Neste manual, outras definições podem gerar dúvidas como, por exemplo, os raticidas que apesar de ser uma das categorias para a classificação do agente tóxico, também são citados como outro grupo importante de agrotóxicos/uso agrícola e uso doméstico. Este fato demonstra que ainda não há homogeneidade das definições e dos critérios adotados para o registro destas notificações. A ocorrência de problemas na classificação do agente tóxico também demonstra que os centros não utilizam a classificação dos produtos saneantes, descrita na legislação sanitária brasileira.

Nos registros estudados constatou-se até nove classificações diferentes para uma mesma categoria de produto, como no caso dos desinfestantes. A classificação deste tipo de produto como medicamento talvez possa ser explicada pelo hábito já consagrado pela população de chamar este tipo de produto de "remédio" (para barata, formiga, cupim, rato). A definição de remédio na língua portuguesa é "aquilo que combate o mal, a dor e a doença” 25 (p. 1.739). Este conceito permite confundir "veneno" (combate o mal) com "remédio" (combate a dor e a doença). Peres 26 constatou este fato com os pesticidas agrícolas e trabalhadores rurais e, justificou o fato como sendo decorrente das diferenças nos padrões 
de comunicação da população rural e urbana, devido, entre outros fatores, ao analfabetismo, ao precário acesso às informações e à educação formal. Entretanto, demonstramos neste estudo que este hábito ocorre também na população urbana e com pessoas de outros níveis de escolaridade, como no caso dos plantonistas dos CCIs que possuem, pelo menos, o grau superior incompleto. A identificação correta, com nome e marca do produto envolvido na intoxicação, é um importante instrumento para dirimir as dúvidas da classificação.

Acrescentando-se à estatística anual, os casos de intoxicação por saneantes registrados com outra classificação nos CCIs, temos um aumento médio de $27,5 \%$ ao ano, no número de casos de intoxicação não intencional com produtos saneantes, sendo que a classificação de produtos químicos industriais é a que mais contribui para este aumento.

A via de exposição foi identificada na maioria dos casos e, como em outros países 14,15, a de maior freqüência foi a ingestão. Para os gerenciadores do risco fica o questionamento: as outras vias de exposição não ocorrem ou os CCIs são procurados apenas para os casos de ingestão?

A identificação do fator determinante daquele evento é imprescindível para que medidas preventivas possam ser implementadas. A informação de que a circunstância foi um acidente individual ou coletivo reflete apenas o número de pessoas acometidas pelo evento, mas não esclarece qual foi a causa do acidente, nem possibilita diferenciar se o mesmo foi desencadeado por uma característica do produto (cor, odor, forma de apresentação, tipo de embalagem), por um comportamento inadequado da população ou por ambos. Esta informação ficou sujeita à anotação aleatória do plantonista do CCI, uma vez que não há um campo de preenchimento obrigatório para este dado.

Os CCIs têm como função principal orientar os cuidados médicos a um intoxicado, entretanto, nos seus registros é possível identificar usos inadequados ou inesperados, circunstâncias e cenários específicos de exposição. Os registros de casos individuais são fontes valiosas destas informações e por isso, a toxicologia clínica tem sido desafiada a contribuir na prevenção de intoxicações e na implementação de ações de saúde pública, melhorando a segurança da população que a cada dia está exposta a uma maior variedade de agentes tóxicos domésticos e ambientais, aumentando, deste modo, o impacto da toxicovigilância na saúde pública 4,27 .

O maior problema de avaliar dados provenientes de diferentes CCIs é a falta de homogeneidade da abordagem dos dados 26,27. Por isso, há necessidade de validação dos dados através da padronização do formato da informação, da seleção consistente de critérios para causas e outros conceitos, que devem ser largamente aceitos pelos especialistas como já acontece na área de farmacovigilância para detecção e avaliação de eventos adversos induzidos por medicamentos. A utilização de critérios que não são consensuais geram informações duvidosas que levam a conclusões errôneas 4,8 .

No Estado do Rio de Janeiro, as intoxicações exógenas foram incluídas na lista de doenças e agravos à saúde, de notificação compulsória através da publicação da Resolução $n^{\circ}$. 1.110, de 30 de janeiro de 1997 28, que foi revogada pela Resolução $n^{\circ}$. 2.075, de 20 de junho de 200329 , sob a alegação de mudanças do perfil epidemiológico dos agravos à saúde da coletividade. Comparando-se as duas resoluções, verificou-se que somente as intoxicações exógenas foram retiradas da listagem, embora os dados existentes nos SINITOX e nos CCIs não demonstrem a ocorrência destas mudanças.

O perfil clínico e as circunstâncias das intoxicações devem ser analisados periodicamente para que medidas preventivas ou corretivas possam ser propostas. Estas informações devem ser geradas considerando a sua importância para a proposição de políticas de saúde e conseqüentemente, para a sociedade. Para isso, a informação deve permitir a identificação do problema e gerar consciência social para o evento estudado.

\section{Conclusão}

As limitações identificadas afetam substancialmente a vigilância de um relevante problema de saúde pública e provavelmente reduzem sua efetividade. A revisão dos processos de coleta, registro e gerenciamento de dados se impõe para corrigir as limitações apontadas. 


\section{Resumo}

Os sistemas de informação em saúde devem ser fonte de informações confiáveis e válidas, para que possam ser utilizadas pelos sistemas de vigilância para reduzir morbidade e mortalidade na população. Intoxicação não intencional é de interesse para a saúde pública, uma vez que é a principal causa de atendimento de emergência pediátrica. As informações sobre estes eventos estão registradas nos Centros de Controle de Intoxicações (CCIs). Este estudo analisou os registros de intoxicações não intencionais com produtos saneantes domissanitários existentes nos dois CCIs do Estado do Rio de Janeiro, Brasil, no período de 2000-2002. As variáveis analisadas foram sexo, idade, causa do acidente, via de exposição, agente tóxico e evolução do caso. Nos 2.810 registros estudados foi identificada a população vulnerável de meninos até quatro anos e a via de exposição (oral). Entretanto, a análise dos dados relativos ao agente tóxico, causa e evolução demonstrou que os dados registrados atualmente nos CCIs são inconsistentes. Há necessidade de revisar as definições adotadas pelos CCIs para que as informações geradas possam ser efetivas em subsidiar ações e políticas de vigilância sanitária.

Envenenamento; Centros de Controle de Intoxicação; Sistemas de Informação; Vigilância Sanitária

\section{Colaboradores}

R. F. Presgrave foi a responsável pelo planejamento, execução do estudo e redação final do texto. L. A. B. Camacho e M. H. S. Villas Boas participaram da revisão final do texto.

\section{Referências}

1. Brasil. Lei nº 8.080, de 19 de setembro de 1990 . Dispõe sobre as condições para a promoção, proteção e recuperação da saúde, a organização e o funcionamento dos serviços correspondentes e dá outras providências. Diário Oficial União 1990; 20 set.

2. Derelanko MJ, Hollinger MA. CRC handbook of toxicology. New York: CRC Press; 1995.

3. Vincoli JW. Risk management for hazardous chemicals. Boca Raton: CRC Press; 1997.

4. Descostes J, Testud F. Toxicovigilance: a new approach for the hazard identification and risk assessment of toxicants in human beings. Toxicol Appl Pharmacol 2005; 207(2 Suppl):599-603.

5. Lessa FJD, Mendes ACG, Farias SF, Sá DA, Duarte PO, Melo Filho DA. Novas metodologias para vigilância epidemiológica: uso do sistema de informações hospitalares - SIH/SUS. Inf Epidemiol SUS 2000; 9 Suppl 1:3-27.
6. Centers for Disease Control and Prevention. Updated guidelines for evaluating public health surveillance systems. http://www.cdc.gov/mmwr/ preview/mmwrhtm/rr5013a1.htm (acessado em 06/Fev/2007).

7. Departamento de Estudos em Ciência e Tecnologia, Centro de Informação Científica e Tecnológica, Fundação Oswaldo Cruz. Manual de preenchimento da ficha de notificação e de atendimento: centros de informação, de controle e de atendimento toxicológico. Rio de Janeiro: Centro de Informação Científica e Tecnológica, Fundação Oswaldo Cruz; 2001.

8. Kearney T, Hiatt P, Olson K. Effectiveness of protocols and clinical guidelines in poison center operations. J Toxicol Clin Toxicol 2001; 39:227.

9. Tempowski J, Persson H. Quality and accreditation of poisons centers. Report of an EAPCCT working group. J Toxicol Clin Toxicol 2001; 39:226. 
10. Persson H, Tempowski J. Developing and maintaining quality in poisons information centers. Toxicology 2004; 198:263-6.

11. Makalinao IR, Awang R. Poison control centers in developing countries and Asia's need for toxicology education. Toxicol Appl Pharmacol 2005; 207 (2 Suppl):716-21.

12. Wilkerson R, Northington L, Fisher W. Ingestion of toxic substances by infants and children: what we don't know can hurt. Crit Care Nurse 2005; 25: 35-44.

13. Berry M. Poisoning. Can Pharm J 1996; 129:19-22.

14. Mena C, Bettini M, Cerda P, Concha F, Paris E. Epidemiology of intoxications in Chile: ten years of registry. Rev Méd Chile 2004; 132:493-9.

15. Litovitz TL, Watson WA, Klein-Schwartz W, Rodgers GC, Youniss J, Reid N, et al. 2003 annual report of the American Association of Poison Control Centers Toxic Exposure Surveillance System. Am J Emerg Med 2004; 22:335-404.

16. Brasil. Decreto n ${ }^{\circ}$. 79.094, de 5 de janeiro de 1977. Regulamenta a Lei $\mathrm{n}^{\circ}$. 6.360, de 23 de setembro de 1976, que submete a sistema de vigilância sanitária os medicamentos, insumos farmacêuticos, drogas, correlatos, cosméticos, produtos de higiene, saneante e outros. Diário Oficial da União 1977; 7 jan.

17. Brasil. Portaria DISAD n ${ }^{\circ}$. 15, de 23 de agosto de 1988. Atualiza o regulamento para o registro de produtos saneantes domissanitários com ação antimicrobiana. Diário Oficial da União 1988; 5 set.

18. Brasil. Portaria SVS no. 89, de 25 de agosto de 1994. Aprova as normas referentes ao registro dos produtos saneantes domissanitários "água sanitária" e "alvejantes", na categoria detergentes e congêneres e desinfetantes. Diário Oficial da União 1994; 26 ago.

19. Brasil. Portaria SVS nº. 322, de 28 de julho de 1997. Aprova as normas referentes ao registro dos produtos para uso em jardinagem amadora. Diário Oficial da União 1997; 5 ago.
20. Brasil. Portaria SVS $n^{\circ}$. 326, de 9 de novembro de 2005. Aprova o regulamento técnico para produtos desinfestantes domissanitários harmonizado no âmbito do MERCOSUL através da Resolução GMC nº. 49/99. Diário Oficial da União 1997; 14 nov.

21. Brasil. Portaria SVS no ${ }^{\circ} .152$, de 26 de fevereiro de 1999. Aprova o regulamento técnico para o registro de produtos destinados à desinfecção de água para o consumo humano e de produtos algicidas e fungicidas para piscinas. Diário Oficial da União 1999; 1 mar.

22. Brasil. Resolução ANVISA no. 117, de 11 de junho de 2001. Estabelece as normas e os procedimentos referentes ao registro de produtos biológicos de uso domissanitário. Diário Oficial da União 2001; 13 jun.

23. Brasil. Resolução ANVISA RDC nº. 240, de 6 de outubro de 2004. Altera os dizeres de rotulagem dos produtos fortemente alcalinos e fortemente ácidos. Diário Oficial da União 2004; 8 out.

24. Santana RAL. Sistema Nacional de Informações Tóxico-Farmacológicas: o desafio da padronização dos dados [Dissertação de Mestrado]. Rio de Janeiro: Escola Nacional de Saúde Pública, Fundação Oswaldo Cruz; 2004.

25. Ferreira ABH. Novo Aurélio século XXI: o dicionário da língua portuguesa. Rio de Janeiro: Editora Nova Fronteira; 1999.

26. Peres F. É veneno ou remédio? Agrotóxicos, saúde e ambiente. Rio de Janeiro: Editora Fiocruz; 2003.

27. Laborde A. New roles for poison control centers in developing countries. Toxicology 2004; 198:273-7.

28. Rio de Janeiro. Resolução no. 1.110 , de 30 de janeiro de 1997. Complementa o quadro de doenças e agravos à saúde de notificação compulsória no Estado do Rio de Janeiro. Diário Oficial do Estado do Rio de Janeiro 1997; 31 jan.

29. Rio de Janeiro. Resolução nº. 2.075, de 20 de junho de 2003. Redefine a relação de doenças de notificação no âmbito do Estado do Rio de Janeiro. Diário Oficial do Estado do Rio de Janeiro 2003; 23 jun.

Recebido em 16/Abr/2007

Versão final reapresentada em 18/Mar/2008

Aprovado em 12/Ago/2008 